\title{
The cohesin subunit Rad21 is a negative regulator of hematopoietic self-renewal through epigenetic repression of HoxA7 and HoxA9
}

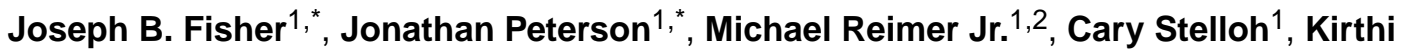 \\ Pulakanti $^{1}$, Zachary J. Gerbec ${ }^{3}$, Alex M. Abel ${ }^{3}$, Jennifer Miksanek Strouse ${ }^{6}$, Christopher \\ Strouse $^{5}$, Maureen McNulty ${ }^{4}$, Subramaniam Malarkannan ${ }^{1,3,5,6}$, John D. Crispino ${ }^{4}$, Samuel \\ Milanovich ${ }^{7}$, and Sridhar Rao ${ }^{1,2,6, \#}$ \\ ${ }^{1}$ Blood Research Institute, BloodCenter of Wisconsin, Milwaukee, WI \\ 2Department of Cell Biology, Neurobiology, and Anatomy, Medical College of Wisconsin, \\ Milwaukee, WI \\ ${ }^{3}$ Department of Microbiology and Molecular Genetics, Medical College of Wisconsin, Milwaukee, \\ WI \\ ${ }^{4}$ Northwestern University Division of Hematology/Oncology, Chicago, IL \\ ${ }^{5}$ Department of Medicine, Medical College of Wisconsin, Milwaukee, WI \\ ${ }^{6}$ Department of Pediatrics, Medical College of Wisconsin, Milwaukee, WI \\ ${ }^{7}$ Sanford Research Center and University of South Dakota Sanford School of Medicine, Sioux \\ Falls, SD
}

\section{Abstract}

Acute myelogenous leukemia (AML) is a high-risk hematopoietic malignancy caused by a variety of mutations, including genes encoding the cohesin complex. Recent studies have demonstrated that reduction in cohesin complex levels leads to enhanced self-renewal in hematopoietic stem and progenitors (HSPCs). We sought to delineate the molecular mechanisms by which cohesin mutations promote enhanced HSPC self-renewal since this represents a critical initial step during leukemic transformation. We verified that RNAi against the cohesin subunit Rad21 causes enhanced self-renewal of HSPCs in vitro through derepression of Polycomb Repressive Complex 2 (PRC2) target genes, including Hoxa7 and Hoxa9. Importantly, knockdown of either Hoxa7 or Hoxa9 suppressed self-renewal, implying both are critical downstream effectors of reduced cohesin levels. We further demonstrate that the cohesin and PRC2 complexes interact and are

Users may view, print, copy, and download text and data-mine the content in such documents, for the purposes of academic research, subject always to the full Conditions of use: http://www.nature.com/authors/editorial_policies/license.html\#terms

\# corresponding author: Sridhar Rao, Blood Research Institute, Blood Center of Wisconsin, 8733 West Watertown Plank Road,

Milwaukee, WI 53226, 414-937-3841 (phone), 414-937-6284 (fax), sridhar.rao@bcw.edu.

These authors contributed equally to this work.

Conflicts of Interest:

All authors declare they have no conflicts of interest to report.

Supplementary Information:

Supplementary information is available at the Leukemia website. 
bound in close proximity to Hoxa7 and Hoxa9. Rad 21 depletion resulted in decreased levels of H3K27me3 at the Hoxa7 and Hoxa9 promoters, consistent with Rad21 being critical to proper gene silencing by recruiting the PRC2 complex. Our data demonstrates that the cohesin complex regulates PRC2 targeting to silence $\mathrm{Hoxa} 7$ and $\mathrm{Hoxa} 9$ and negatively regulate self-renewal. Our studies identify a novel epigenetic mechanism underlying leukemogenesis in AML patients with cohesin mutations.

\section{Introduction}

Acute Myelogenous Leukemia (AML) is an aggressive myeloid malignancy ${ }^{1,2}$. Even when patients are treated with highly intense chemotherapy regimens, including consolidation with allogeneic bone marrow transplantation (BMT), long-term survival rates remain poor. The cause of this poor prognosis for patients with AML is multifactorial. One common finding hindering the development of novel therapeutics is high genetic variability among patients ${ }^{3}$. A subset of patients have recurrent chromosomal translocations which generate driver oncogenic fusion proteins such as RUNX1-RUNX1T1 or MYH11-CBFB'; however, the majority of patients do not have a clear structural abnormality driving their leukemia. Many karyotypically normal AML patients have mutations in genes including FLT3, TET2, NPM1, CEBPA, or DNMT3A. A study in which 200 patients with de novo AML were sequenced $^{3}$ revealed that patients have on average 13 distinct somatic mutations within the coding regions of $>250$ well-annotated genes, indicating AML arises from a diverse combination of mutations. A subset of the genes uncovered were not previously described in AML patients including subunits of the cohesin complex (RAD21, STAG2, SMC1A, $S M C 3)^{3-7}$. Cohesin mutations tend to occur early within disease pathogenesis ${ }^{3,6}$ implying that these mutations are critical events during leukemia development. Subsequent studies have confirmed that cohesin mutations are relatively common in AML, and frequently cooccur with known driver mutations including NPM1 and FLT3-ITD ${ }^{3,4,6-8}$.

Complete loss of cohesin function causes an embryonic lethal phenotype in knock-out mice, implying that some level of cohesin function is absolutely required for normal development ${ }^{9-12}$. Interestingly, somatic cohesin mutations in AML are uniformly heterozygous and not associated with dramatic changes in chromosomal number, implying that cohesin mutations promote AML through a function independent of their role during mitosis $^{3,6,7,13,14}$. Cohesin plays a critical role in controlling gene expression ${ }^{13,15,16}$. The complex facilitates DNA looping that permits interactions between distal genomic segments and gene promoters facilitating transcriptional regulation in cis $^{17-19}$. The cohesin complex interacts with CCCTC-binding factor (CTCF) to organize the genome and recruit cis regulatory elements which promote gene silencing ${ }^{17,20-22}$. Given the importance of transcriptional regulators in the pathogenesis of $\mathrm{AML}^{3}$, it has been hypothesized that cohesin mutations promote AML through altered gene expression.

Recent studies demonstrate that reduced cohesin expression confers enhanced self-renewal on hematopoietic stem and progenitor cells (HSPCs) both in vitro and in vivo ${ }^{23-26}$. These studies demonstrated that reducing expression of single cohesin genes induces genome-wide changes in chromatin accessibility, and when combined with Flt3-internal tandem 
duplications (ITD) mutations it resulted in potentiated Stat5 signaling and 100\% penetrant leukemia with an average latency of 6 months ${ }^{24}$. Other studies observed altered numbers of higher order hematopoietic stem and progenitor cells (LT- and ST-HSCs) in vivo, after prolonged cohesin-depletion ${ }^{23-25}$, whereas more differentiated progenitor populations (CMP, GMP, MEP) remained unchanged ${ }^{23,25}$. Prolonged cohesin-depletion causes increased HSPC self-renewal, a potential mechanism to leukemic transformation ${ }^{23,26}$. Thus, while multiple groups have demonstrated cohesin mutations promote enhanced HSPC self-renewal and altered gene expression, the precise molecular mechanism connecting cohesin depletion to these changes remains unclear.

\section{Materials and Methods}

A detailed Materials and Methods section can be found in the supplemental material.

\section{Results}

\section{Rad21 depletion augments self-renewal and proliferation of murine HSPCs in vitro}

Rad21 is a critical component of the cohesin complex and to define its role in normal hematopoiesis in vitro, we transduced murine HSPCs with puromycin-resistance encoding lentiviruses containing two independent shRNAs targeted to non-overlapping regions of Rad21. RT-qPCR and immunoblotting confirmed Rad21 mRNA (Figure S1A and 1A) and protein (Figure 1B, C) was efficiently depleted in shRNA treated samples 24 hours after viral transduction and after primary and secondary passages in methylcellulose.

To assess whether Rad21 depletion altered self-renewal in vitro, we performed serial replating assays ${ }^{23-26}$. Through serial replating, $\operatorname{Rad} 21$-depleted cells maintained the ability to generate CFUs, and showed enhanced proliferation (Figure 1D, E). During the fourth replating Rad21-depleted colonies were larger and denser than control colonies (Figure 1F). Based on recent findings that chronic cohesin depletion in HSPCs causes elevated erythroid potential $^{25}$, we monitored granulocyte/marcophage (CFU-GM), granulocyte/erythrocyte/ monocyte/megakaryocyte (CFU-GEMM), and erythroid blast forming unit (CFU-BFU-E) colonies at the end of primary methylcellulose passage. We observed no statistically significant differences between the Rad21-depleted HSPCs compared to controls (Figure 1G). From these data we conclude that Rad 21 depletion confers enhanced self-renewal to murine HSPCs in vitro consistent with published studies ${ }^{23-26}$.

One possible explanation for the enhanced self-renewal is that Rad 21 depletion selectively depletes lower order progenitors, thereby enriching for short term- or long termhematopoietic stem cells (LT-HSCs, ST-HSCs). Thus, we monitored percentages of c-kitnegative/lineage-positive and c-kit-positive/lineage-negative cells, and observed no significant differences between the Rad21-depleted and empty vector cells (Figure S2A and S2B). Importantly, this assay is not sensitive enough to distinguish between subtypes of higher order HSPCs (LT-HSCs, ST-HSCs, and MPPs). These data are consistent with previous studies which observed minimal changes in differentiation of cohesin-deficient HSPCs at 1 week during ex vivo culture ${ }^{26}$. 


\section{Rad21 depletion does not induce aneuploidy}

Given that patients with germline heterozygous cohesin mutations are not predisposed to aneuploidy ${ }^{11,27}$, we hypothesized that $\operatorname{Rad} 21$ depletion of $50 \%$ would not induce aneuploidy ${ }^{28}$. We identified cells in $\mathrm{G}_{0} / \mathrm{G}_{1}$ (2N DNA content), S-phase ( $\left.2 \mathrm{~N}-4 \mathrm{~N}\right)$, Mitosis $(4 \mathrm{~N})$, and aneuploid cells $(<2 \mathrm{~N}$ or $>4 \mathrm{~N})$ by flow cytometry. In accordance with published studies ${ }^{7,23-26}$, we observed no significant increases in aneuploid cells following Rad21 depletion, but did observe enhanced self-renewal evidenced by decreased cells in $\mathrm{G}_{0} / \mathrm{G}_{1}$ with increased cells in S and M-phase following Rad21 depletion (Figure S3).

\section{Rad21 depletion causes increased expression of the self-renewal factor Hoxa9}

We hypothesized that $R a d 21$ depletion increases self-renewal through alterations in gene expression. Next, we isolated mRNA from HSPCs at the end of the primary passage and conducted targeted transcriptome analyses by microarray. Overall, approximately 1,000 probes ( 384 probes UP and 660 DOWN) demonstrated a minimum 2-fold change following Rad21 depletion, consistent with reduced Rad 21 levels causing broad changes in gene expression (Table S5). Among genes with a statistically significant change following Rad21 depletion (Figure 2A and Table S5), we noted an approximately 10-fold increase in the expression of Hoxa9, a homeodomain-containing transcription factor that is over-expressed in about half of human AMLs. HoxA9 expression is associated with a poor prognosis ${ }^{29-31}$ and is known to confer enhanced self-renewal on HSPCs in vivo and in vitro ${ }^{32,33}$. We reasoned that if Rad21/cohesin negatively regulates $H o x a 9$, its expression would be elevated rapidly and chronically following $\operatorname{Rad} 21$ depletion. We noted a statistically significant increase in $H_{o x a 9}$ expression within 24 hours of $\operatorname{Rad} 21$ depletion (Figure 2B), which was maintained through secondary replatings (Figure 2C). Next we reanalyzed microarray data sets in which a 4-hydroxytamoxifen (4-OHT)-inducible Hoxa9 expression vector was used $^{34}$ (GSE21299) to identify genes activated (Hoxa9-Up) or repressed (Hoxa9-Down) by Hoxa9 overexpression. We analyzed Hoxa9-altered genes using Gene Set Enrichment Analysis (GSEA) to determine if they demonstrated a statistically significant, concordant difference with the gene lists generated from Rad21-depleted $\mathrm{HSPCs}^{35}$. We observed a significant concordance with Rad21 depletion (Figure 3A), specifically that Hoxa9-activated genes were elevated and Hoxa9-repressed genes were repressed following Rad21 depletion. To confirm that a small subset of genes was not driving this phenomenon, we selected the top 100 genes increased or decreased by Hoxa9 overexpression and monitored changes in the same probes after Rad 21 depletion. Additionally, we compared our microarray data to a recently published RNA-seq data set in which cohesin subunits ( $\operatorname{Rad} 21$ and Smc3) were depleted and found that in all samples, Hoxa9 target genes showed a similar pattern of expression $^{25}$ (Figure 3B). More broadly we found substantial overlap between our microarray data and previously published RNA-seq data ${ }^{25}$ (Figure S4).

Given the increased self-renewal phenotype we wondered whether Meis1, which cooperates with Hoxa9 to induce AMLs, was also overexpressed by Rad21 depletion ${ }^{36-38}$. RT-qPCR analyses did not reveal any differences in Meis 1 expression (Figure S5). Collectively, these data show that $\mathrm{Rad} 21$ depletion induces aberrant expression of $\mathrm{Hoxa} 9$ and its downstream targets, but has no effect on Meis1 expression, which is consistent with the observation that cohesin depletion alone is insufficient to drive leukemic transformation. 


\section{Additional HoxA family members exhibit increased expression in Rad21-depleted HSPCs}

Because the entire Hoxa locus is silenced by PRC2 during hematopoiesis ${ }^{21,39}$, we speculated that additional Hoxa factors would exhibit elevated expression. Thus, we reanalyzed published RNA-seq data from cohesin-depleted HSPCs ${ }^{25}$, and found elevated expression of Hoxa7 and Hoxa9, with smaller changes in other HoxA genes (Figure S6A). We confirmed these data by RT-qPCR and included Hoxb4 as a negative control since it was unchanged following cohesin depletion ${ }^{25}$ (Figure S6B). Given the known role of the cohesin complex in modulating expression of genes within chromatin domains ${ }^{40,41}$, these data imply that $\operatorname{Rad} 21$ is required to repress the chromatin domain containing Hoxa7 and Hoxa9.

\section{AML patients with cohesin mutations have elevated levels of Hoxa 7 and $\mathrm{Hoxa} 9$}

Given changes in $\mathrm{Hoxa}$ and $\mathrm{Hoxa}_{9}$ expression following cohesin depletion, we hypothesized that AML patients harboring cohesin mutations would express higher levels of both $H O X A 7$ and $H O X A 9$ compared to AML patients lacking cohesin mutations. Next, we reanalyzed transcriptome datasets from the Cancer Genome Atlas (TCGA), of which $47 \%$ were karyotypically normal ${ }^{3,4}$. Among patients with (25) or without (172) cohesin mutations (SMC1A, SMC3, STAG2, or RAD21) we observed a statistically significant increase in both HOXA 7 and HOXA9 expression in cohesin-mutated AML (Figure 3C). Consistent with a multiple hit model of AML, cohesin mutations co-occur most frequently with mutations in NPM1 (p-value $6.32 \times 10^{-33,4}$ ).

\section{Depletion of Hoxa7 or Hoxa9 diminshes HSPC self-renewal}

Given the elevated expression of Hoxa7 and Hoxa9, we wondered whether depletion of either of these two factors could rescue the enhanced self-renewal phenotype elicited by Rad21 depletion. Thus, we transduced HSPCs with empty vector alone, Rad21-shRNAs alone, or Rad21-shRNAs combined with Hoxa9- or Hoxa7-shRNAs. At the end of primary methylcellulose plating we observed fewer colonies present in the Rad21/Hoxa7 or Rad21/ Hoxa9 co-depleted cultures (Figure S7A, Figure S8A). This reduction in colony number was associated with overall reduced cell numbers, and the phenotype was maintained in secondary platings (Figure S7B, C, D, Figure S8B, C, D). These data are consistent with previously published reports highlighting a role of Ноха7 and Ноха 9 in regulating HSPC self-renewal ${ }^{37,42-44}$. Importantly, depletion of $\mathrm{Hoxa}$ or Hoxa9 alone (Figure S9A-B) diminishes HSPC self-renewal (Figure S9C-G). Thus, both $\mathrm{Hoxa} 7$ and $\mathrm{Hoxa} 9$ are required for HSPC self-renewal, and their effects following Rad21 depletion are not a form of epistasis.

\section{Rad21 cooperates with PRC2 to repress target gene expression}

Previous studies have shown that Hoxa7 and Hoxa9 expression are repressed by the PRC2 complex $^{39,45}$, and selectively induced in myeloid- and erythroid-committed progenitor cells by removal of its associated histone mark $\mathrm{H} 3 \mathrm{~K} 27 \mathrm{me} 3^{46,47}$. To identify pathways altered by Rad21 depletion in an unbiased fashion, we performed GSEA and screened 3,395 different pathways (gene sets). Each curated gene set was generated by perturbing a biological system with different chemical, RNAi, or genetic means. We narrowed the list (Table S6) by focusing on gene sets derived from hematopoietic tissues and noted an upregulation of 38 
genes repressed by Ezh2 (Figure 4A), the catalytic subunit of the PRC2 complex ${ }^{48}$. We confirmed this derepression by a differential expression analysis (Figure 4B). Collectively, these data imply that Rad 21 depletion causes derepression of PRC2 target genes.

The derepression of a large number of its target genes suggests either reduced PRC2 levels or impaired activity. To investigate the former we performed immunoblotting for two members of the PRC2 complex and observed no significant difference in the levels of Ezh2 or Suz12 (Figure 4C-E). Next, we assessed if global levels of histone H3K27me3 were altered by $\operatorname{Rad} 21$ depletion. Following $R a d 21$ depletion we observed an approximately $40 \%-50 \%$ reduction in histone H3K27me3 levels (Figure 4C\&F). From these data we conclude that Rad21 depletion reduces levels of the repressive mark H3K27me3 without disrupting PRC2 expression.

\section{Cohesin and PRC2 complexes interact in murine myeloid cells}

Given our results, we hypothesized that cohesin and PRC2 interact to mediate epigenetic silencing. First, we reanalyzed a published proteomics study that characterized binding partners of the PRC2 complex ${ }^{49}$ and noted the core PRC2 subunit Eed interacted with Rad21 in two murine cell types (Figure S10). Next, we performed co-immunoprecipitation experiments using lysates from immortalized murine myeloblast cells (32D cells). We observed interactions between members of the cohesin and PRC2 complexes, and could identify these interactions by immunoprecipitating either complex (Figure 5A). Members of the cohesin and PRC2 complexes are shown in Figure 5B for reference. Whether the interactions observed represent direct or indirect association between the two complexes will require additional studies.

\section{Rad21 mediates PRC2 recruitment to the Hoxa9 locus and deposition of H3K27me3}

Given that Rad21 and PRC2 interact in myeloid cells and that Hoxa genes are silenced by $\mathrm{PRC}^{30,45}$, we hypothesized that cohesin would be bound near the Hoxa7 and Hoxa9 locus. Using data from both the ENCODE projects, we identified Rad21/RAD21 occupancy between the Hoxa7/HOXA7 and Hoxa9/HOXA9 genes at a Ctcf/CTCF binding site. The entire HoxalHOXA locus exhibits high levels of enrichment of the repressive histone mark H3K27me3 consistent with PRC2-mediated silencing. These patterns were observed in both mouse (Figure S11) and human (Figure S12) hematopoietic and non-hematopoietic cell lines. The proximity of a cohesin:CTCF site was intriguing; in other contexts CTCF mediates gene silencing, and in the Hoxa cluster facilitates PRC2 targeting ${ }^{17,21,22}$. Next, we hypothesized that $R a d 21$ depletion induces $H o x A 7 \& 9$ expression through loss of $\mathrm{H} 3 \mathrm{~K} 27 \mathrm{me}$. ChIP analyses revealed a significant reduction in H3K27me3 at the promoters of both Hoxa 7 and Hoxa9 following Rad21 depletion (Figure 6A). In summary, these data demonstrate that Rad21 facilitates PRC2-mediated silencing of Hoxa7 and Hoxa9 (Figure $6 \mathrm{~B})$.

Given the colocalization of cohesin, PRC2, and CTCF at the Hoxa locus (Figure S11 and S12) we wondered whether depletion of Ctcf in HSPCs would phenocopy the enhanced selfrenewal observed following Rad21 depletion. Ctcf depletion had minimal effect on Hoxa7 (Figure S13B) or Hoxa9 (Figure S13C) expression. The lack of elevated Hoxa 7 and Hoxa9 
expression, was accompanied by a reduced number of CFUs and cells numbers (Figures S13D, E). These data suggest that although CTCF likely plays a role in regulating PRC2 and cohesin occupancy at the Hoxa locus its functions are so diverse that its depletion is not tolerated by HSPCs.

\section{Discussion}

AML is a complex myeloid malignancy hallmarked by genetic heterogeneity, and as with most cancers, multiple cooperating mutations are involved in oncogenic transformation. This implies that different genetic mutations disrupt similar pathways inducing a shared phenotype such as enhanced self-renewal. In this study, we focused on cohesin mutations, which are frequently observed in AML. Recent studies have shown that reduced cohesin promotes hematopoietic self-renewal, and when combined with FLT3-ITD causes 100\% penetrant $\mathrm{AML}^{23-26}$. In addition, these studies observed changes in chromatin accessibility following cohesin depletion. In one study, genes displaying increased chromatin accessibility around their TSS demonstrated increased occupancy by transcription factors critical to self-renewal such as Gata2, Erg, and Runx $1^{23}$. Our study lends further mechanistic, in that the PRC2 histone mark H3K27me3 classically promotes recruitment of the PRC1 complex which mediates polynucleosome compaction ${ }^{50,51}$. Thus, cohesin loss may ultimately impart enhanced self-renewal by preventing proper chromatin compaction around genes that need to be silenced for proper differentiation.

One open question is how the cohesin complex is targeted to the Hoxa locus to recruit the PRC2 complex to mediate epigenetic silencing. One possible explanation is that cohesin regulates gene expression by modulating genomic architecture. Key insights into the role of cohesin in regulating gene expression were uncovered with the discovery of a direct interaction between cohesin and the DNA binding protein $\mathrm{CTCF}^{17}$. CTCF plays a central role in regulating gene expression at multiple levels, including acting as an insulator to block enhancer:gene interactions or to organize the genome into topologically associated domains (TADs ${ }^{52-55}$ ). The interaction between CTCF and cohesin was initially thought to be critical in regulating genomic architecture, specifically establishment of TADs. Interestingly, seminal studies from the Merkenschlager lab demonstrated that homozygous deletion of Rad21 in non-cycling thymocytes does not alter TAD architecture, but rather disrupts longrange interactions within TAD compartments (sub-TADs ${ }^{41}$ ). The authors found that TAD architecture overall remained intact, but that cohesin loss resulted in compression of the dynamic range of gene expression through loss of sub-TAD interactions. This is interesting because other studies have shown that the Hoxa cluster is regulated in a tissue-specific manner by CTCF and cis-regulatory elements located within sub-TADs surrounding the Hoxa locus which ultimately results in PRC2-mediated gene silencing ${ }^{21,56-58}$ Our data supports a model whereby cohesin is essential for proper PRC2 recruitment and inhibition of Hoxa 7 and Hoxa9 expression, which may be the result of a sub-TAD interaction dependent upon cohesin. Unlike the results of Seitan et $\mathrm{a}^{41}$, we did not see broad increases across all Hox clusters, as would be predicted if all sub-TAD interactions were disrupted. The discrepancy is likely secondary to differences in the level of cohesin loss, specifically our use of an approximately 50\% reduction in $\operatorname{Rad} 21$ levels versus complete deletion of $\operatorname{Rad} 21$. Alternatively, it may be secondary to our use of cycling bone marrow cells, as opposed to 
non-cycling thymocytes. Importantly, this raises the intriguing hypothesis that some cohesindependent chromatin interactions require a higher level of cohesin than others.

Our data demonstrates an interaction between the cohesin and PRC2 complexes, disruption of which increases expression of the self-renewal factor Hoxa9. Determining whether the interactions between the cohesin and PRC2 complexes are direct or indirect will require additional studies. Modulators of this pathway could provide possible therapeutic targets for patients with cohesin-mutated AML. Hoxa9 expression has been shown to be, in part, activated by di- or tri-methylation of histone $\mathrm{H} 3$ on lysine 79 (H3K79me2/me3) catalyzed by Dot11, which is blocked by PRC2 activity ${ }^{59-61}$. Dot11 inhibitors are already in clinical trials for MLL rearranged AML; therefore, these could provide a possible therapeutic approach that could reverse the aberrant expression of Hoxa genes in cohesin-mutated AML. Whether or not these histone marks are altered upon Rad21/cohesin depletion remains unknown, but provide testable hypotheses to identify therapeutic targets for patients with cohesin-mutated AML.

\section{Supplementary Material}

Refer to Web version on PubMed Central for supplementary material.

\section{Acknowledgments}

The authors would like to thank Hope Campbell and Benedetta Bonacci for their assistance with flow cytometry. S. Rao is supported by grants from the Midwest Athletes Against Childhood Cancer (MACC Fund), Hyundai Hope on Wheels, and an American Society of Hematology Bridge Grant. Additional research support to S. Rao provided by charitable gifts from Hartland Blood Centers, Ms. Nanette Gardetto, and Mr. Doug Ziegler.

\section{References}

1. Burnett A, Wetzler M, Lowenberg B. Therapeutic Advances in Acute Myeloid Leukemia. Journal of Clinical Oncology. 2011; 29:487-494. [PubMed: 21220605]

2. Thakar MS, Talano J-AM, Tower RL, Kelly ME, Burke MJ. Indications for transplantation in childhood acute leukemia and the impact of minimal residual disease on relapse: a review. Clinical Practice. 2014; 11:79-90.

3. Cancer Genome Atlas Research Network. Genomic and epigenomic landscapes of adult de novo acute myeloid leukemia. N Engl J Med. 2013; 368:2059-2074. [PubMed: 23634996]

4. Welch JS, Ley TJ, Link DC, Miller CA, Larson DE, Koboldt DC, et al. The origin and evolution of mutations in acute myeloid leukemia. Cell. 2012; 150:264-278. [PubMed: 22817890]

5. Kon A, Shih L-Y, Minamino M, Sanada M, Shiraishi Y, Nagata Y, et al. Recurrent mutations in multiple components of the cohesin complex in myeloid neoplasms. Nat Genet. 2013; 45:12321237. [PubMed: 23955599]

6. Thota S, Viny AD, Makishima H, Spitzer B, Radivoyevitch T, Przychodzen B, et al. Genetic alterations of the cohesin complex genes in myeloid malignancies. Blood. 2014; 124:1790-1798. [PubMed: 25006131]

7. Thol F, Bollin R, Gehlhaar M, Walter C, Dugas M, Suchanek KJ, et al. Mutations in the cohesin complex in acute myeloid leukemia: clinical and prognostic implications. Blood. 2014; 123:914920. [PubMed: 24335498]

8. Garg M, Nagata Y, Kanojia D, Mayakonda A, Yoshida K, Haridas Keloth S, et al. Profiling of somatic mutations in acute myeloid leukemia with FLT3-ITD at diagnosis and relapse. Blood. 2015; 126:2491-2501. [PubMed: 26438511] 
9. Xu H, Balakrishnan K, Malaterre J, Beasley M, Yan Y, Essers J, et al. Rad21-Cohesin Haploinsufficiency Impedes DNA Repair and Enhances Gastrointestinal Radiosensitivity in Mice. PLoS ONE. 2010; 5:e12112. [PubMed: 20711430]

10. Remeseiro S, Cuadrado A, pez GGOM-LO, Pisano DG, Losada A. A unique role of cohesin-SA1 in gene regulation and development. EMBO J. 2012; 31:2090-2102. [PubMed: 22415368]

11. Kawauchi S, Calof AL, Santos R, Lopez-Burks ME, Young CM, Hoang MP, et al. Multiple Organ System Defects and Transcriptional Dysregulation in the Nipbl+/ Mouse, a Model of Cornelia de Lange Syndrome. PLoS Genet. 2009; 5:e1000650. [PubMed: 19763162]

12. White JK, Gerdin A-K, Karp NA, Ryder E, Buljan M, Bussell JN, et al. Genome-wide generation and systematic phenotyping of knockout mice reveals new roles for many genes. Cell. 2013; 154:452-464. [PubMed: 23870131]

13. Leeke B, Marsman J, O'Sullivan JM, Horsfield JA. Cohesin mutations in myeloid malignancies: underlying mechanisms. Experimental Hematology \& Oncology. 2014; 3:13. [PubMed: 24904756]

14. Yoshida K, Toki T, Okuno Y, Kanezaki R, Shiraishi Y, Sato-Otsubo A, et al. The landscape of somatic mutations in Down syndrome-related myeloid disorders. Nat Genet. 2013; 45:1293-1299. [PubMed: 24056718]

15. Mehta GD, Kumar R, Srivastava S, Ghosh SK. Cohesin: Functions beyond sister chromatid cohesion. FEBS Letters. 2013; 587:2299-2312. [PubMed: 23831059]

16. Williams MS, Somervaille TCP. Leukemogenic Activity of Cohesin Rings True. Cell Stem Cell. 2015; 17:642-644. [PubMed: 26637939]

17. Wendt KS, Yoshida K, Itoh T, Bando M, Koch B, Schirghuber E, et al. Cohesin mediates transcriptional insulation by CCCTC-binding factor. Nature. 2008; 451:796-801. [PubMed: 18235444]

18. Kagey MH, Newman JJ, Bilodeau S, Zhan Y, Orlando DA, van Berkum NL, et al. Mediator and cohesin connect gene expression and chromatin architecture. Nature. 2010; 467:430-435. [PubMed: 20720539]

19. Merkenschlager M, Odom DT. CTCF and Cohesin: Linking Gene Regulatory Elements with Their Targets. Cell. 2013; 152:1285-1297. [PubMed: 23498937]

20. Baranello L, Kouzine F, Levens D. CTCF and cohesin cooperate to organize the 3D structure of the mammalian genome. Proc Natl Acad Sci USA. 2014; 111:889-890. [PubMed: 24398527]

21. Xu M, Zhao G-N, Lv X, Liu G, Wang LY, Hao D-L, et al. CTCF controls HOXA cluster silencing and mediates PRC2-repressive higher-order chromatin structure in NT2/D1 cells. Molecular and Cellular Biology. 2014; 34:3867-3879. [PubMed: 25135475]

22. Li T, Hu J-F, Qiu X, Ling J, Chen H, Wang S, et al. CTCF regulates allelic expression of Igf2 by orchestrating a promoter-polycomb repressive complex 2 intrachromosomal loop. Molecular and Cellular Biology. 2008; 28:6473-6482. [PubMed: 18662993]

23. Mazumdar C, Shen Y, Xavy S, Zhao F, Reinisch A, Li R, et al. Leukemia-Associated Cohesin Mutants Dominantly Enforce Stem Cell Programs and Impair Human Hematopoietic Progenitor Differentiation. Cell Stem Cell. 2015; 17:675-688. [PubMed: 26607380]

24. Viny AD, Ott CJ, Spitzer B, Rivas M, Meydan C, Papalexi E, et al. Dose-dependent role of the cohesin complex in normal and malignant hematopoiesis. Journal of Experimental Medicine. 2015; 212:1819-1832. [PubMed: 26438361]

25. Mullenders J, Aranda-Orgilles B, Lhoumaud P, Keller M, Pae J, Wang K, et al. Cohesin loss alters adult hematopoietic stem cell homeostasis, leading to myeloproliferative neoplasms. Journal of Experimental Medicine. 2015; 212:1833-1850. [PubMed: 26438359]

26. Galeev R, Baudet A, Kumar P, Rundberg Nilsson A, Nilsson B, Soneji S, et al. Genome-wide RNAi Screen Identifies Cohesin Genes as Modifiers of Renewal and Differentiation in Human HSCs. Cell Reports. 2016; 14:2988-3000. [PubMed: 26997282]

27. Castronovo P, Gervasini C, Cereda A, Masciadri M, Milani D, Russo S, et al. Premature chromatid separation is not a useful diagnostic marker for Cornelia de Lange syndrome. Chromosome Res. 2009; 17:763-771. [PubMed: 19690971]

28. Liu J, Krantz ID. Cornelia de Lange syndrome, cohesin, and beyond. Clinical Genetics. 2009; 76:303-314. [PubMed: 19793304] 
29. Golub TR, Slonim DK, Tamayo P, Huard C, Gaasenbeek M, Mesirov JP, et al. Molecular Classification of Cancer: Class Discovery and Class Prediction by Gene Expression Monitoring. Science. 1999; 286:531-537. [PubMed: 10521349]

30. Spencer DH, Young MA, Lamprecht TL, Helton NM, Fulton R, O'Laughlin M, et al. Epigenomic analysis of the HOX gene loci reveals mechanisms that may control canonical expression patterns in AML and normal hematopoietic cells. Leukemia. 2015; 29:1279-1289. [PubMed: 25600023]

31. Li Z, Zhang Z, Li Y, Arnovitz S, Chen P, Huang H, et al. PBX3 is an important cofactor of HOXA9 in leukemogenesis. Blood. 2013; 121:1422-1431. [PubMed: 23264595]

32. Kroon E, Krosl J, Thorsteinsdottir U, Baban S, Buchberg AM, Sauvageau G. Hoxa9 transforms primary bone marrow cells through specific collaboration with Meis1a but not Pbx1b. EMBO J. 1998; 17:3714-3725. [PubMed: 9649441]

33. Thorsteinsdottir U, Mamo A, Kroon E, Jerome L, Bijl J, Lawrence HJ, et al. Overexpression of the myeloid leukemia-associated Hoxa9 gene in bone marrow cells induces stem cell expansion. Blood. 2002; 99:121-129. [PubMed: 11756161]

34. Muntean AG, Tan J, Sitwala K, Huang Y, Bronstein J, Connelly JA, et al. The PAF Complex Synergizes with MLL Fusion Proteins at HOX Loci to Promote Leukemogenesis. Cancer Cell. 2010; 17:609-621. [PubMed: 20541477]

35. Subramanian A, Tamayo P, Mootha VK, Mukherjee S, Ebert BL, Gillette MA, et al. Gene set enrichment analysis: a knowledge-based approach for interpreting genome-wide expression profiles. Proc Natl Acad Sci USA. 2005; 102:15545-15550. [PubMed: 16199517]

36. Lawrence HJ, Rozenfeld S, Cruz C, Matsukuma K. Frequent co-expression of the HOXA9 and MEIS1 homeobox genes in human myeloid leukemias. Leukemia. 1999

37. Afonja O, Smith JE, Cheng DM, Goldenberg AS, Amorosi E, Shimamoto T, et al. MEIS1 and HOXA7 genes in human acute myeloid leukemia. Leukemia Research. 2000; 24:849-855. [PubMed: 10996203]

38. Thorsteinsdottir U, Kroon E, Jerome L, Blasi F, Sauvageau G. Defining Roles for HOX and MEIS1 Genes in Induction of Acute Myeloid Leukemia. Molecular and Cellular Biology. 2001; 21:224 234. [PubMed: 11113197]

39. Bracken AP, Dietrich N, Pasini D, Hansen KH, Helin K. Genome-wide mapping of Polycomb target genes unravels their roles in cell fate transitions. Genes Dev. 2006; 20:1123-1136. [PubMed: 16618801]

40. Seitan VC, Hao B, Tachibana-Konwalski K, Lavagnolli T, Mira-Bontenbal H, Brown KE, et al. A role for cohesin in T-cell-receptor rearrangement and thymocyte differentiation. Nature. 2011; 476:467-471. [PubMed: 21832993]

41. Seitan VC, Faure AJ, Zhan Y, McCord RP, Lajoie BR, Ing-Simmons E, et al. Cohesin-based chromatin interactions enable regulated gene expression within preexisting architectural compartments. Genome Research. 2013; 23:2066-2077. [PubMed: 24002784]

42. Ayton PM. Transformation of myeloid progenitors by MLL oncoproteins is dependent on Hoxa7 and Hoxa9. Genes Dev. 2003; 17:2298-2307. [PubMed: 12952893]

43. Kroon E, Thorsteinsdottir U, Mayotte N, Nakamura T, Sauvageau G. NUP98-HOXA9 expression in hemopoietic stem cells induces chronic and acute myeloid leukemias in mice. EMBO J. 2001; 20:350-361. [PubMed: 11157742]

44. Faber J, Krivtsov AV, Stubbs MC, Wright R, Davis TN, van den Heuvel-Eibrink M, et al. HOXA9 is required for survival in human MLL-rearranged acute leukemias. Blood. 2009; 113:2375-2385. [PubMed: 19056693]

45. Nagel S, Venturini L, Marquez VE, Meyer C, Kaufmann M, Scherr M, et al. Polycomb repressor complex 2 regulates HOXA9 and HOXA10, activating ID2 in NK/T-cell lines. Mol Cancer. 2010; 9:151. [PubMed: 20565746]

46. Sauvageau G, Lansdorp PM, Eaves CJ, Hogge DE, Dragowska WH, Reid DS, et al. Differential expression of homeobox genes in functionally distinct CD34+ subpopulations of human bone marrow cells. Proc Natl Acad Sci USA. 1994; 91:12223-12227. [PubMed: 7527557]

47. Lawrence HJ. Loss of expression of the Hoxa-9 homeobox gene impairs the proliferation and repopulating ability of hematopoietic stem cells. Blood. 2005; 106:3988-3994. [PubMed: 16091451] 
48. Kamminga LM, Bystrykh LV, de Boer A, Houwer S, Douma J, Weersing E, et al. The Polycomb group gene Ezh2 prevents hematopoietic stem cell exhaustion. Blood. 2006; 107:2170-2179. [PubMed: 16293602]

49. Cao Q, Wang X, Zhao M, Yang R, Malik R, Qiao Y, et al. The central role of EED in the orchestration of polycomb group complexes. Nat Commun. 2014; 5:3127. [PubMed: 24457600]

50. Di Croce L, Helin K. Transcriptional regulation by Polycomb group proteins. Nat Struct Mol Biol. 2013; 20:1147-1155. [PubMed: 24096405]

51. Simon JA, Kingston RE. Occupying Chromatin: Polycomb Mechanisms for Getting to Genomic Targets, Stopping Transcriptional Traffic, and Staying Put. Mol Cell. 2013; 49:808-824. [PubMed: 23473600]

52. Dixon JR, Selvaraj S, Yue F, Kim A, Li Y, Shen Y, et al. Topological domains in mammalian genomes identified by analysis of chromatin interactions. Nature. 2012; 485:376-380. [PubMed: 22495300]

53. Sanyal A, Lajoie BR, Jain G, Dekker J. The long-range interaction landscape of gene promoters. Nature. 2013; 489:109-113.

54. Ong C-T, Corces VG. CTCF: an architectural protein bridging genome topology and function. Nat Rev Genet. 2014; 15:234-246. [PubMed: 24614316]

55. Phillips JE, Corces VG. CTCF: master weaver of the genome. Cell. 2009; 137:1194-1211. [PubMed: 19563753]

56. Berlivet S, Paquette D, Dumouchel A, Langlais D, Dostie J, Kmita M. Clustering of tissue-specific sub-TADs accompanies the regulation of HoxA genes in developing limbs. PLoS Genet. 2013; 9:e1004018. [PubMed: 24385922]

57. Vieux-Rochas M, Fabre PJ, Leleu M, Duboule D, Noordermeer D. Clustering of mammalian Hox genes with other H3K27me3 targets within an active nuclear domain. Proceedings of the National Academy of Sciences. 2015; 112:4672-4677.

58. Noordermeer D, Leleu M, Schorderet P, Joye E, Chabaud F, Duboule D. Temporal dynamics and developmental memory of 3D chromatin architecture at Hox gene loci. Elife. 2014; 3:e02557. [PubMed: 24843030]

59. Bernt KM, Zhu N, Sinha AU, Vempati S, Faber J, Krivtsov AV, et al. MLL-Rearranged Leukemia Is Dependent on Aberrant H3K79 Methylation by DOT1L. Cancer Cell. 2011; 20:66-78. [PubMed: 21741597]

60. Okada Y, Feng Q, Lin Y, Jiang Q, Li Y, Coffield VM, et al. hDOT1L Links Histone Methylation to Leukemogenesis. Cell. 2005; 121:167-178. [PubMed: 15851025]

61. Chen S, Yang Z, Wilkinson AW, Deshpande AJ, Sidoli S, Krajewski K, et al. The PZP Domain of AF10 Senses Unmodified H3K27 to Regulate DOT1L-Mediated Methylation of H3K79. Mol Cell. 2015; 60:319-327. [PubMed: 26439302] 
A

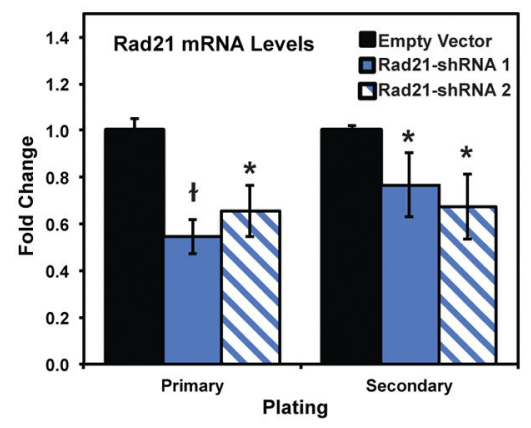

D

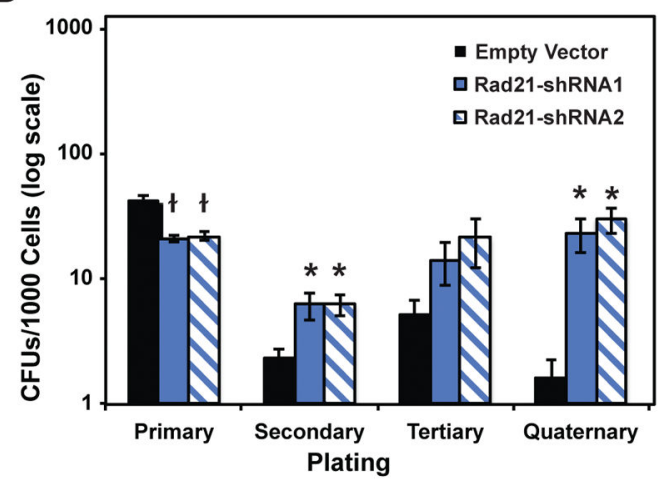

$\mathbf{F}$

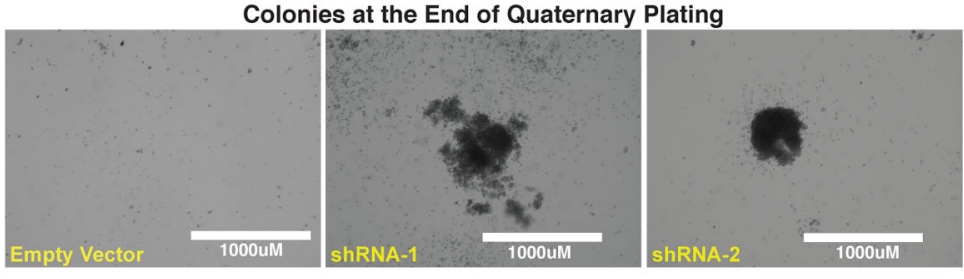

C

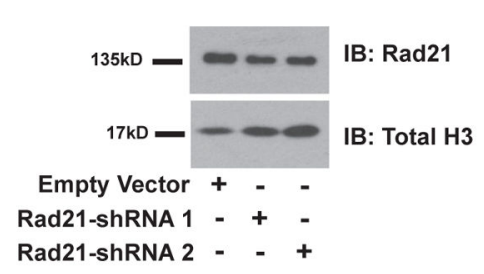

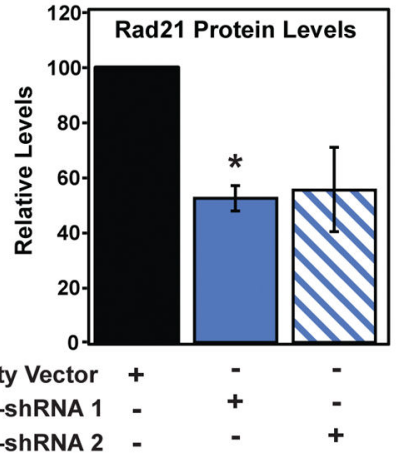

E

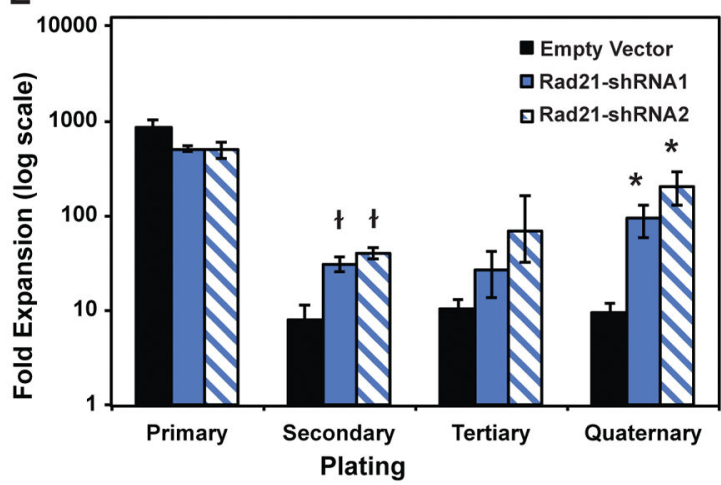

G End of Primary Plating

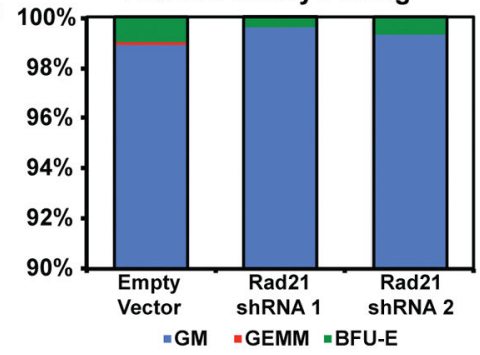

Figure 1. Rad21 depletion enhances self-renewal of primary HSPCs

(A) qRT-PCR analysis of control and Rad21-depleted lineage negative HSPCs at the end of primary and secondary methylcellulose platings. (B) Immunoblotting of Rad21-depleted and control HSPCs using antibodies against Rad21 and Total Histone H3. (C) Densitometry of the blot shown in panel B. (D+E) Control and Rad21-depleted HSPCs were serially passaged in methylcellulose and after each passage colony number (D: CFUs/1000 cells) and total cell numbers (E: fold expansion) were determined. (F) Representative images of colonies from empty vector and Rad21-depleted HSPCs at the end of quaternary plating. (G) Colony counts (GM, BFU-E, GEMM) of empty vector and Rad21-depleted HSPCs at the end of primary methylcellulose culture. 
A

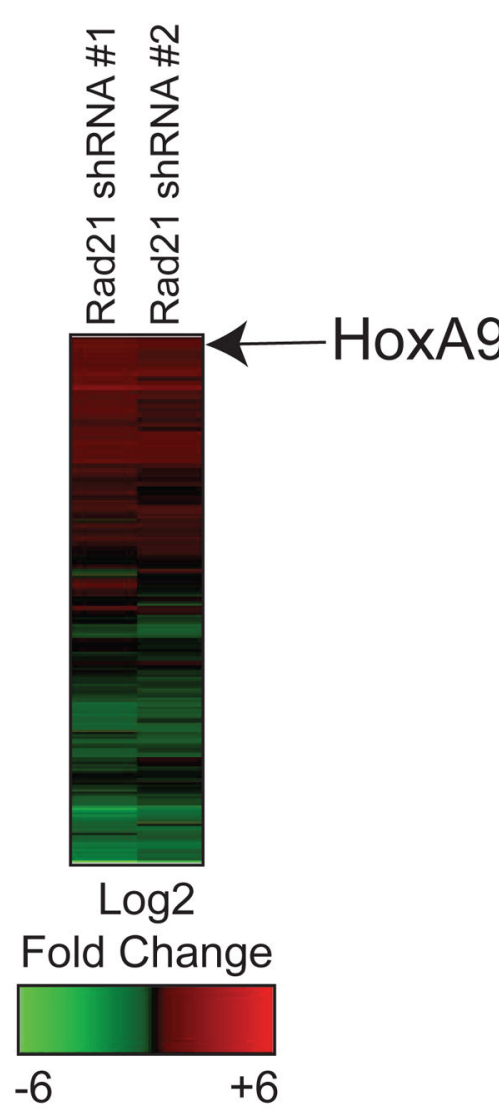

B
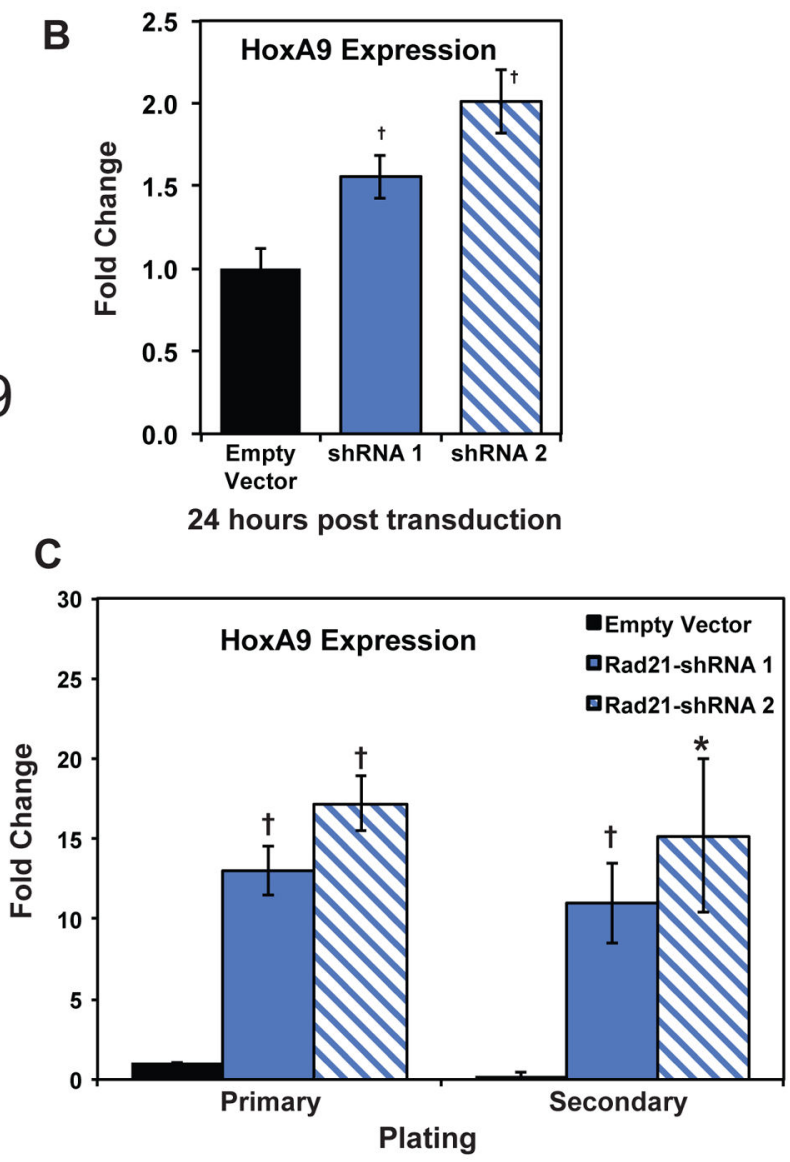

Figure 2. Rad21 depletion increases Hoxa9 expression

(A) Microarray heat map showing the gene expression changes following Rad21 depletion. (B+C) qRT-PCR analysis of empty vector and Rad21-depleted HSPCs after (B) 24 hours, or (C) after culture in methylcellulose. 
A

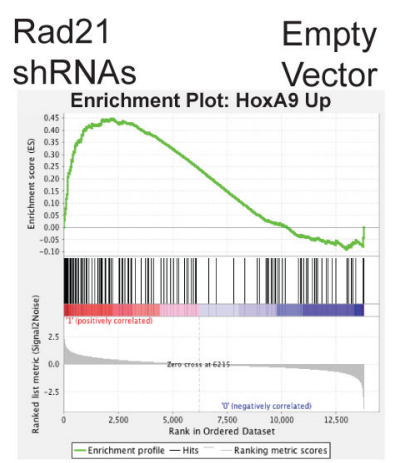

NES: +1.84

p-val: $<0.001$

FDR: $<0.01 \%$
Rad21 Empty

ShRNAs Vector

Enrichment Plot: HoxA9 Down
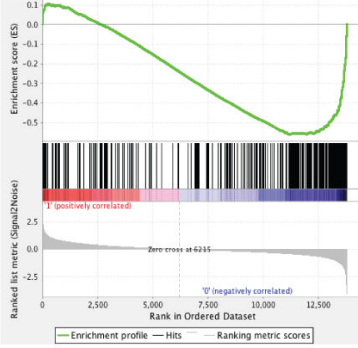

NES:-2.52

p-val: $<0.001$

FDR: $<0.01 \%$

C

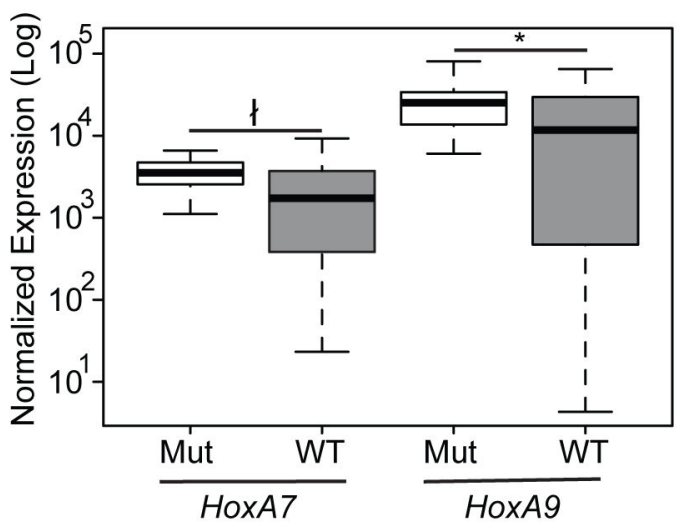

Rad21, Smc1a, Smc3, or Stag2 mut $(n=25)$

Rad21, Smc1a, Smc3, or Stag2 wt $(\mathrm{n}=172)$

B

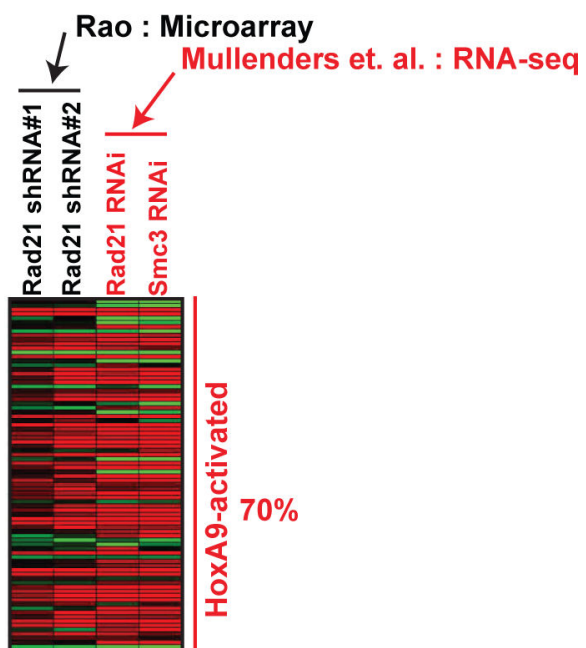

Figure 3. Rad21 depletion alters expression of Hoxa9 target genes

(A) GSEA was performed comparing control and Rad21-depleted HSPCs against a gene set compiled from ${ }^{34}$ consisting of genes that exhibit increased (Hoxa9 Up) or decreased (Hoxa9 Down) expression following forced Hoxa9 expression. (B) Heat map showing fold changes of Rad21-shRNA1 and shRNA2 over empty vector using the list of genes in Panel A. RNAsequencing data from ${ }^{25}$ was included for comparison, in which either Rad21 or $S m c 3$ were depleted. The percent concordance between the microarray and RNA-seq datasets, is shown on the right. (C) Hoxa7 and Hoxa9 probes were analyzed from cohesin-mutant and cohesinWT AML patients. () indicates \# of patients. 
A

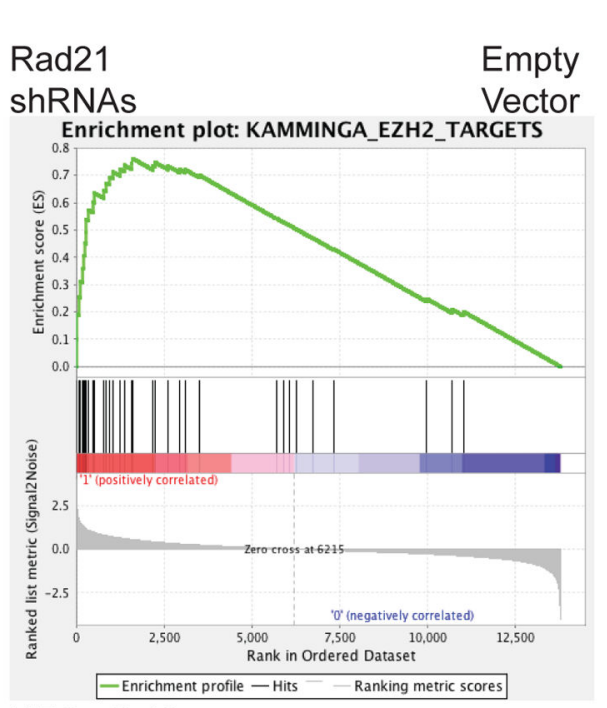

NES:+2.49

p-val: $<0.001$

FDR: $<0.1 \%$

D

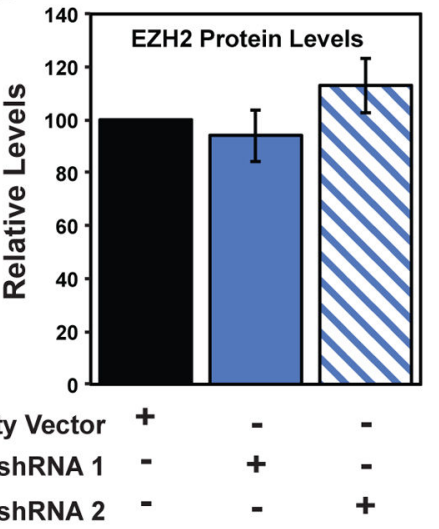

B
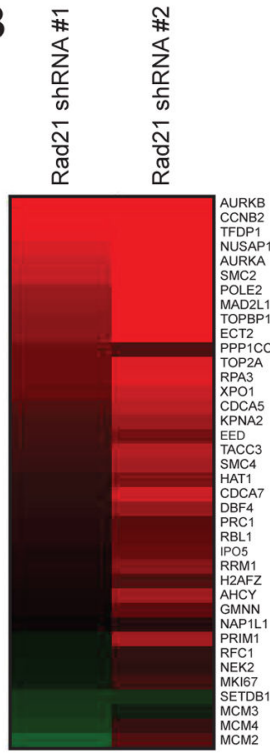

Log2 Fold Change

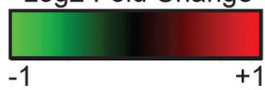

C

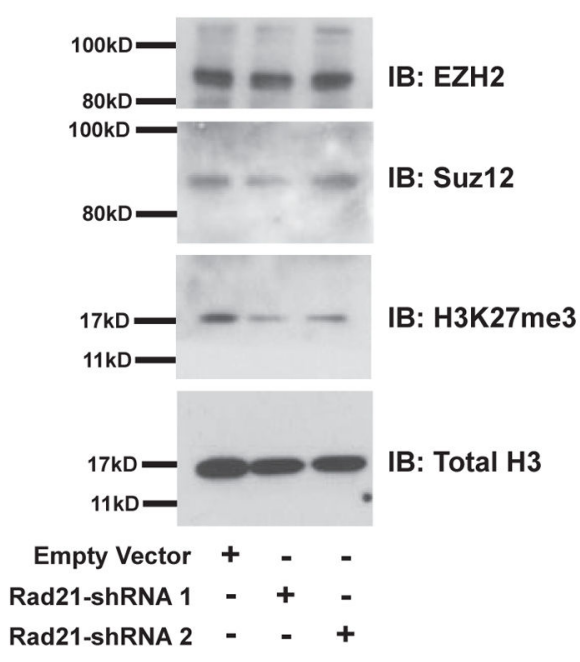

E

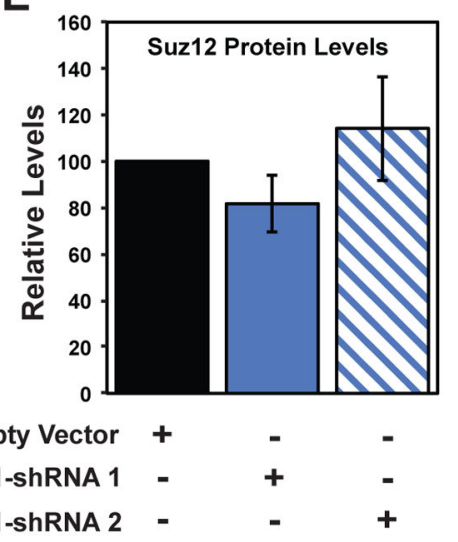

$\mathbf{F}$

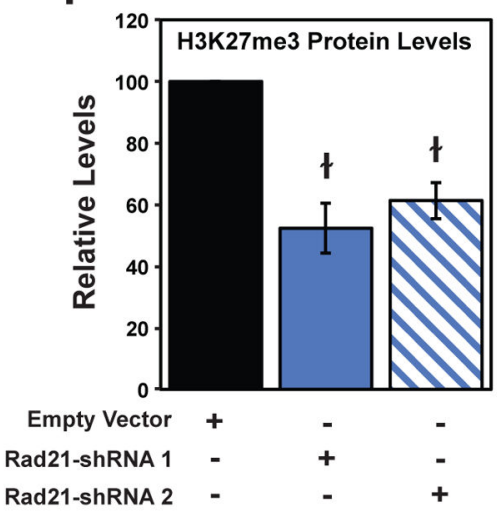

Figure 4. Rad21 depletion causes de-repression of PRC2 target genes (A) GSEA showing the correlation between Rad21-depleted HSPCs and genes repressed by the PRC2 subunit Ezh2. (B) Heat map showing increased expression of Ezh2-repressed genes. (C) Immunoblot analysis of lysates from empty vector and Rad21-depleted cells at the end of primary methylcellulose plating. (D-F) Densitometry of blots shown in panel C showing quantitation of 3 independent biological replicates for Ezh2 (D), Suz12 (E), and H3K27me3 (F). 
A

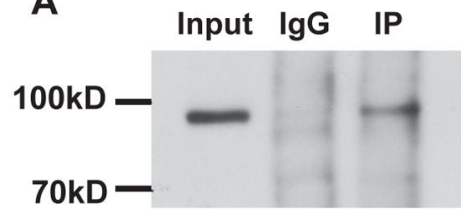

IP: Smc3

IB: Suz12
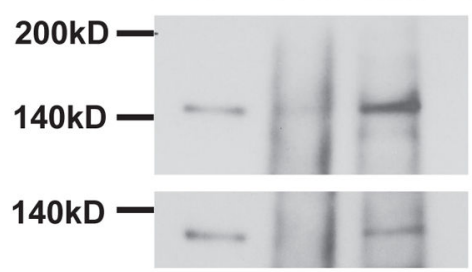

$140 \mathrm{kD}-$

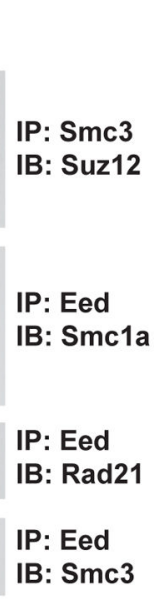

B $\quad$ PRC2
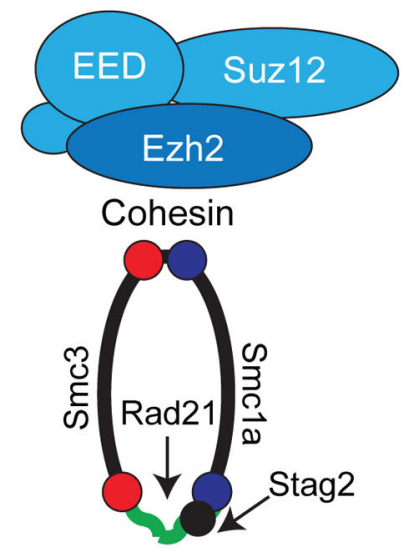

Figure 5. Cohesin and PRC2 interact in murine myeloid cells

(A) Endogenous immunoprecipitation from mouse myeloid progenitor cells. Precipitated proteins were analyzed by western blotting using antibodies against various cohesin and PRC2 complex members. (B) Schematic of the cohesin and PRC2 complexes. 

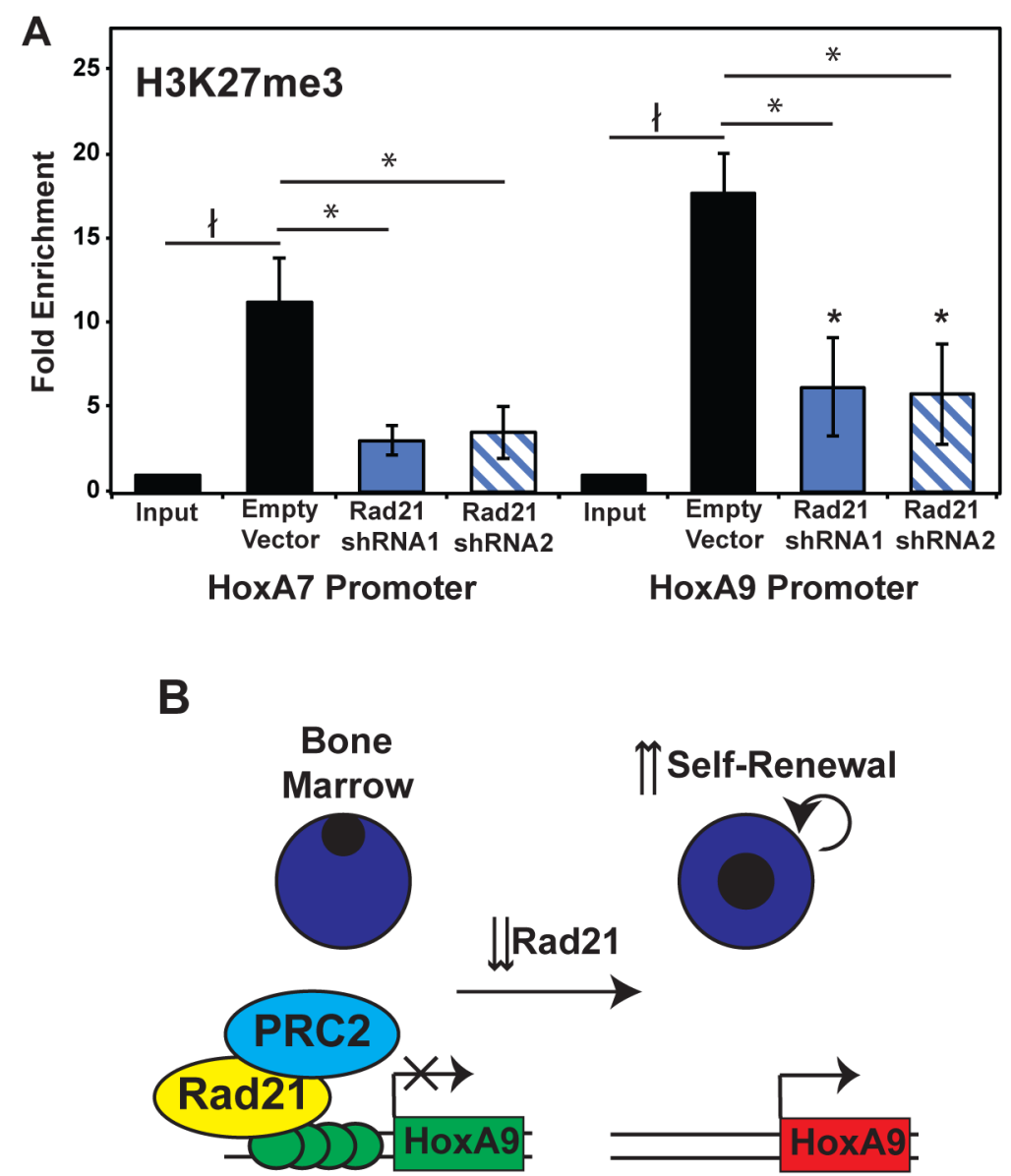

\section{H3K27me3}

Figure 6. Rad21 depletion decreases histone H3K27me3 deposition at the Hoxa9 locus (A) ChIP was performed on control and Rad21-shRNA1 and shRNA2 HSPCs at the end of primary methylcellulose culture. Chromatin was precipitated using an antibody against histone H3K27me3 and purified DNA was analyzed by qPCR using primers against the Hoxa 7 and Hoxa9 promoters. Each data point represents 6 and 3 independent biological replicates for Hoxa7 and Hoxa9 respectively. (B) Model for how Rad21 regulates expression of Hoxa7 and Hoxa9. 